\title{
Tangible UI by Object and Material Classification with Radar
}

\author{
Hui-Shyong Yeo \\ University of St Andrews \\ hsy@st-andrews.ac.uk
}

\author{
Barrett Ens \\ University of South Australia \\ barrett.ens@unisa.edu.au
}

\author{
Aaron Quigley \\ University of St Andrews \\ aquigley@st-andrews.ac.uk
}
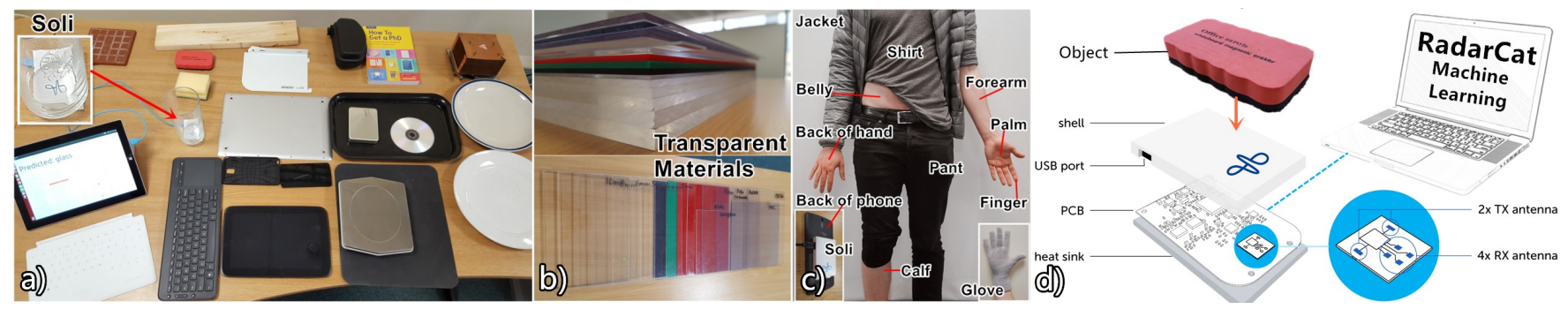

Figure 1: Recognizing a) everyday objects b) transparent materials c) different body parts and d) setup (From [Yeo et al. 2016])

\begin{abstract}
Radar signals penetrate, scatter, absorb and reflect energy into proximate objects and ground penetrating and aerial radar systems are well established. We describe a highly accurate system based on a combination of a monostatic radar (Google Soli), supervised machine learning to support object and material classification based UIs. Based on RadarCat techniques, we explore the development of tangible user interfaces without modification of the objects or complex infrastructures. This affords new forms of interaction with digital devices, proximate objects and micro-gestures.
\end{abstract}

\section{CCS CONCEPTS}

•Human-centered computing $\rightarrow$ Interaction techniques; Interactive systems and tools; Ubiquitous and mobile devices;

\section{KEYWORDS}

Radar sensing, tangible interaction, object recognition

\section{ACM Reference format:}

Hui-Shyong Yeo, Barrett Ens, and Aaron Quigley. 2017. Tangible UI by Object and Material Classification with Radar. In Proceedings of SA '17 Emerging Technologies, Bangkok,Thailand, November 27-30, 2017, 2 pages. DOI: $10.1145 / 3132818.3132824$

\section{INTRODUCTION}

Visions of computing including Tangible User Interfaces (TUI), context awareness are "Discreet Computing" are predicated on knowledge of their environments being employed to adapt the user experience. In context awareness, systems can adapt according to their location of use, the collection of nearby people and objects, as well as changes to these objects over time. While TUI attempt to give digital information a physical and manipulable form. RadarCat [Yeo et al. 2016] advances our ability to realize such visions with radar based sensing. It allows for new forms and styles of

SA '17 Emerging Technologies, Bangkok,Thailand

2017. 978-1-4503-5404-2/17/11.

DOI: $10.1145 / 3132818.3132824$ interaction, and entirely new classes of user interface to emerge, including discreet ones.

To understand one's physical environment, one needs to understand and know about the objects present. Computer vision techniques, which require the object to be visible, well lit and within range for a suitable resolution have been explored in a range of object tracking approaches such as sensing surface colours and materials [Yeo et al. 2017]. However, vision based classification suffers from being limited to the material qualities which are present on the surface of the object. This can result in confusion where a layer of opaque material (packaging) blocks the primary object of interest for classification. RadarCat, by contrast, provides a degree of surface level penetration, allowing us to explore materials and objects without being limited to just what is visible on the surface.

Radar systems have been used to recognize particular types of aircraft or materials in luggage or body scanners. Ground-penetrating radar can be used to detect buried objects such as utility pipes or bones. Further non-destructive methods such as near-infrared (NIR) spectroscopy are often utilized for analyzing pharmaceutical products. Likewise, millimeter wave and terahertz technology are being used to detect materials from a distance for scientific exploration or security purposes.

Our goal is to overcome the limitations of camera-based systems, with an embedded sensor that can detect surface material at high speed and accuracy. When the object is placed on or touched by the sensor, the near and fixed distance and incident angle makes our classification task straightforward and allowing for accurate classification. The final design of RadarCat incorporates the following unique capabilities and aspects (a) non-destructive, non-tagging (b) use with surface materials, composite objects and certain body parts and (c) identification of new sensing applications and practical use cases. RadarCat opens up new opportunities in areas such as navigation and world knowledge, consumer interaction, industrial automation, recycling or laboratory process control.

\section{IMPLEMENTATION}

RadarCat uses a Google Soli [Lien et al. 2016] and our software has two parts i) a GUI in C++ using Qt and ii) a classifier backend in 

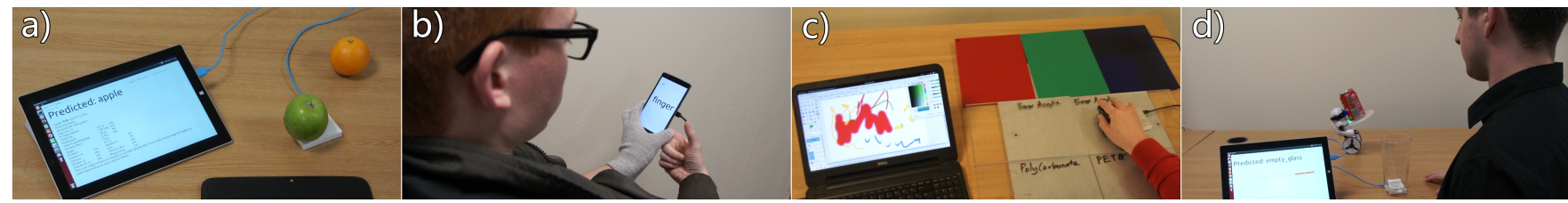

Figure 2: Four example applications to demonstrate the interaction possibilities of RadarCat, from left to right a) physical object dictionary b) tangible painting app c) context-aware interaction and body shortcuts d) automatic refill.

Java using the Weka API. Employing machine learning techniques on the Soli signals, we demonstrate the reliable extraction of data about the target material or object that we use for novel interaction.

Reflected radar signals from many points both within and on the object surface are overlapping and hence contribute to the received signal. As the signals are stable and highly discriminative, we use all 8 channels as input features and extract statistical features, resulting in a total of 661 features to train a Random Forest classifier.

We conducted multiple studies, based on existing study designs, to evaluate several facets of RadarCat on classifying i) everyday materials and objects ii) transparent materials and iii) body parts. Our results, as detailed in the RadarCat paper [Yeo et al. 2016], show high accuracy and robustness.

\section{EXAMPLE USE SCENARIOS}

There are many immediate applications that RadarCat can support. Here we demonstrate four example applications with different interaction possibilities (see figure 2 and video figure). Following this, we speculate about future applications and use cases.

\subsection{Current Applications}

3.1.1 Physical object dictionary. When an object is placed on the sensor (figure 2a), RadarCat recognizes the object and searches for relevant information. This can offer the name in your native language, model number (if man-made), pronunciation, origin, usage, synonyms and equivalent words in a different language.

3.1.2 Context-aware interaction and body shortcuts. A phone with RadarCat embedded (figure 2b) can tell whether it is held in bare palm or in palm wearing a glove. This allows the phone to render larger on-screen buttons to accommodate the fat finger problem when wearing glove. It also supports placement awareness (on desk, sofa or inside pocket) and situation awareness, allowing mode switching automatically. Touching different body parts can activate different shortcut commands instantly placed in a shoulder strap on the upper arm triggers a music/jogging app.

3.1.3 Painting and photo editing application. RadarCat can be used as a physical probe instrument (figure 2c) to quickly and intuitively change the operating mode (scale, rotate, pan) or the brush (size, color, style) based on what the probe is sensing, such as plastics of different material, color and thickness.

3.1.4 Automatic refill. In a restaurant scenario, where RadarCat sensors are embedded in table, we can sense whether a cup is empty or filled, and automatically alert the waiter for refill, all without intervention by the user (figure 2d).

\subsection{Future Applications}

3.2.1 Shopping and self-checkout system. Labelling can be difficult with different types of objects which do not easily lend themselves to marking due to their nature, shape, size or fragility, e.g., vegetables, flowers or eggs. With RadarCat, such objects can be classified for use in both a shop or kitchen.

3.2.2 Assisting those with low vision. While one's sense or touch and smell can mitigate many of the challenges faced with low vision, RadarCat once embedded in gloves or shoes can enhance ones understanding of the proximate environment. Tactile paving on the sidewalk or braille interfaces might be replaced in the future.

3.2.3 Smart medical devices. Current digital medical devices such as thermometers or ultrasound still require the operator to manually note the different body parts being measured. With RadarCat embedded we can allow the automatic tagging of recorded temperature or images with the body part as it is measured.

\section{CONCLUSION AND FUTURE WORK}

Our technique can be used independently or combined with other sensing approaches. This can improve sensing and afford new mobile, wearable and context-aware user interfaces.

The materials scattering and absorption properties at various wavelengths should be investigated further. We further wish to empirically validate the observed ability to recognize different fruits or credit cards, or counting the number of poker cards, or differentiating liquid content in a container. Finally, we would like to explore ways to encode information into an object, or stacking multiple layers of different materials which can be sensed.

\section{REFERENCES}

Jaime Lien, Nicholas Gillian, M. Emre Karagozler, Patrick Amihood, Carsten Schwesig, Erik Olson, Hakim Raja, and Ivan Poupyrev. 2016. Soli: Ubiquitous Gesture Sensing with Millimeter Wave Radar. ACM Trans. Graph. 35, 4, Article 142 (July 2016), 19 pages. DOI : http://dx.doi.org/10.1145/2897824.2925953

Hui-Shyong Yeo, Gergely Flamich, Patrick Schrempf, David Harris-Birtill, and Aaron Quigley. 2016. RadarCat: Radar Categorization for Input \& Interaction. In Proceedings of the 29th Annual Symposium on User Interface Software and Technology (UIST '16). ACM, New York, NY, USA, 833-841. DOI : http://dx.doi.org/10.1145/2984511. 2984515

Hui-Shyong Yeo, Juyoung Lee, Andrea Bianchi, David Harris-Birtill, and Aaron Quigley. 2017. SpeCam: Sensing Surface Color and Material with the Front-facing Camera of a Mobile Device. In Proceedings of the 19th International Conference on HumanComputer Interaction with Mobile Devices and Services (MobileHCI '17). ACM, New York, NY, USA, Article 25, 9 pages. DOI : http://dx.doi.org/10.1145/3098279.3098541 